\title{
Euler-Lagrange Type Cubic Operators and Their Norms on $X_{\curlywedge}$ Space
}

\author{
Abbas Najati ${ }^{1}$ and Asghar Rahimi \\ ${ }^{1}$ Department of Mathematics, Faculty of Sciences, University of Mohaghegh Ardabili, \\ P.O. Box 56199-11367 Ardabili, Iran \\ ${ }^{2}$ Department of Mathematics, University of Maragheh, P.O. Box 55181-83111, Maragheh, \\ East Azarbayjan, Iran \\ Correspondence should be addressed to Abbas Najati, a.najati@uma.ac.ir \\ Received 17 April 2008; Accepted 1 July 2008 \\ Recommended by Jong Kim \\ We will introduce linear operators and obtain their exact norms defined on the function spaces $X_{\curlywedge}$ \\ and $Z_{\lambda}^{5}$. These operators are constructed from the Euler-Lagrange type cubic functional equations \\ and their Pexider versions. \\ Copyright (C) 2008 A. Najati and A. Rahimi. This is an open access article distributed under the \\ Creative Commons Attribution License, which permits unrestricted use, distribution, and \\ reproduction in any medium, provided the original work is properly cited.
}

\section{Introduction}

Let $X$ and $Y$ be complex normed spaces. For a fixed nonnegative real number $\lambda$, we denote by $X_{\curlywedge}$ the linear space of all functions $f: X \rightarrow Y$ (with pointwise operations) for which there exists a constant $M_{f} \geq 0$ with

$$
\|f(x)\| \leq M_{f} e^{\lambda\|x\|}
$$

for all $x \in X$. It is easy to show that the space $X_{\curlywedge}$ with the norm

$$
\|f\|:=\sup _{x \in X}\left\{e^{-\lambda\|x\|}\|f(x)\|\right\}
$$

is a normed space. Let us denote by $X_{\lambda}^{n}$ the linear space of all functions $\underbrace{\phi: X \times \cdots \times X}_{n \text { times }} \rightarrow Y$ (with pointwise operations) for which there exists a constant $M_{\phi} \geq 0$ with

$$
\left\|\phi\left(x_{1}, \ldots, x_{n}\right)\right\| \leq M_{\phi} e^{\lambda \sum_{i=1}^{n}\left\|x_{i}\right\|}
$$


for all $x_{1}, \ldots, x_{n} \in X$. It is not difficult to show that the space $X_{\lambda}^{n}$ with the norm

$$
\|\phi\|:=\sup _{x_{1}, \ldots, x_{n} \in X}\left\{\left\|\phi\left(x_{1}, \ldots, x_{n}\right)\right\| e^{-\lambda \sum_{i=1}^{n}\left\|x_{i}\right\|}\right\}
$$

is a normed space.

We denote by $Z_{\lambda}^{m}$ the normed space $\bigoplus_{i=1}^{m} X_{\curlywedge}=\left\{\left(f_{1}, \ldots, f_{m}\right): f_{1}, \ldots, f_{m} \in X_{\curlywedge}\right\}$ (with pointwise operations) together with the norm

$$
\left\|\left(f_{1}, \ldots, f_{m}\right)\right\|:=\max \left\{\left\|f_{1}\right\|, \ldots,\left\|f_{m}\right\|\right\} .
$$

The norms of the Pexiderized Cauchy, quadratic, and Jensen operators on the function space $X_{\mathcal{\Lambda}}$ have been investigated by Czerwik and Dlutek [1, 2]. In [3], Moslehian et al. have extended the results of [2] to the Pexiderized generalized Jensen and Pexiderized generalized quadratic operators on the function space $X_{\curlywedge}$ and provided more general results regarding their norms.

In [4], Jung investigated the norm of the cubic operator on the function space $Z_{\lambda}^{5}$.

A function $f: X \rightarrow Y$ is called a cubic function if and only if $f$ is a solution function of the cubic functional equation

$$
f(x+y)+f(x-y)=2 f\left(\frac{1}{2} x+y\right)+2 f\left(\frac{1}{2} x-y\right)+12 f\left(\frac{1}{2} x\right) \text {. }
$$

Jun and Kim [5] proved that when both $X$ and $Y$ are real vector spaces, a function $f: X \rightarrow Y$ satisfies (1.6) if and only if there exists a function $B: X \times X \times X \rightarrow Y$ such that $f(x)=B(x, x, x)$ for all $x \in X$, and $B$ is symmetric for each fixed one variable and is additive for fixed two variables.

In [6], the authors introduced the following Euler-Lagrange-type cubic functional equation, which is equivalent to (1.6),

$$
f(x+y)+f(x-y)=a f\left(\frac{1}{a} x+y\right)+a f\left(\frac{1}{a} x-y\right)+2 a\left(a^{2}-1\right) f\left(\frac{1}{a} x\right)
$$

for fixed integers $a$ with $a \neq 0, \pm 1$. Moreover, Jun and Kim [7] introduced the following EulerLagrange-type cubic functional equation

$$
f\left(\frac{1}{a} x+\frac{1}{b} y\right)+f\left(\frac{1}{b} x+\frac{1}{a} y\right)=(a+b)(a-b)^{2}\left[f\left(\frac{1}{a b} x\right)+f\left(\frac{1}{a b} y\right)\right]+a b(a+b) f\left(\frac{1}{a b} x+\frac{1}{a b} y\right)
$$

for fixed integers $a, b$ with $a, b \neq 0, a \pm b \neq 0$, and they proved the following theorem.

Theorem 1.1 (see [7, Theorem 2.1]). Let $X$ and $Y$ be real vector spaces. If a mapping $f: X \rightarrow Y$ satisfies the functional equation (1.6), then $f$ satisfies the functional equation (1.8).

We will introduce linear operators which are constructed from the Euler-Lagrange-type cubic and the Pexiderization of the Euler-Lagrange-type cubic functional equations (1.7) and (1.8). 
Definition 1.2. The operators $C_{1}^{P}, C_{2}^{P}: Z_{\lambda}^{5} \rightarrow X_{\lambda}^{2}$ are defined by

$$
\begin{aligned}
C_{1}^{P}\left(f_{1}, \ldots, f_{5}\right)(x, y):= & f_{1}(x+y)+f_{2}(x-y)-m f_{3}\left(\frac{1}{m} x+y\right) \\
& -m f_{4}\left(\frac{1}{m} x-y\right)-2 m\left(m^{2}-1\right) f_{5}\left(\frac{1}{m} x\right) \\
C_{2}^{P}\left(f_{1}, \ldots, f_{5}\right)(x, y):= & f_{1}\left(\frac{1}{a} x+\frac{1}{b} y\right)+f_{2}\left(\frac{1}{b} x+\frac{1}{a} y\right)-(a+b)(a-b)^{2}\left[f_{3}\left(\frac{1}{a b} x\right)+f_{4}\left(\frac{1}{a b} y\right)\right] \\
& -a b(a+b) f_{5}\left(\frac{1}{a b} x+\frac{1}{a b} y\right)
\end{aligned}
$$

where $a, b$, and $m$ are fixed integers with $a, b \neq 0, a \pm b \neq 0$, and $m \neq 0, \pm 1$.

Definition 1.3. The operators $C_{1}, C_{2}: X_{\curlywedge} \rightarrow X_{\lambda}^{2}$ are defined by

$$
\begin{aligned}
C_{1}(f)(x, y):= & f(x+y)+f(x-y)-m f\left(\frac{1}{m} x+y\right) \\
& -m f\left(\frac{1}{m} x-y\right)-2 m\left(m^{2}-1\right) f\left(\frac{1}{m} x\right), \\
C_{2}(f)(x, y):= & f\left(\frac{1}{a} x+\frac{1}{b} y\right)+f\left(\frac{1}{b} x+\frac{1}{a} y\right) \\
& -(a+b)(a-b)^{2}\left[f\left(\frac{1}{a b} x\right)+f\left(\frac{1}{a b} y\right)\right]-a b(a+b) f\left(\frac{1}{a b} x+\frac{1}{a b} y\right),
\end{aligned}
$$

where $a, b$, and $m$ are fixed integers with $a, b \neq 0, a \pm b \neq 0$, and $m \neq 0, \pm 1$.

In this paper, we will give the exact norms of the operators $C_{1}^{P}, C_{2}^{P}$ on the function space $Z_{\lambda}^{5}$, and norms of the operators $C_{1}, C_{2}$ on the function space $X_{\curlywedge}$. The results extend the results of $[4]$.

\section{Main results}

Throughout this section, $a, b$, and $m$ are fixed integers with $a, b \neq 0, a \pm b \neq 0$, and $m \neq 0, \pm 1$.

The next theorems give us the exact norms of operators $C_{1}^{P}, C_{2}^{P}, C_{1}$, and $C_{2}$.

Theorem 2.1. The operator $C_{1}^{P}: Z_{\lambda}^{5} \rightarrow X_{\lambda}^{2}$ is a bounded linear operator with

$$
\left\|C_{1}^{P}\right\|=2|m|^{3}+2 .
$$

Proof. First, we show that $\left\|C_{1}^{P}\right\| \leq 2|m|^{3}+2$. Since

$$
\max \left\{\|x+y\|,\|x-y\|,\left\|\frac{1}{m} x+y\right\|,\left\|\frac{1}{m} x-y\right\|,\left\|\frac{1}{m} x\right\|\right\} \leq\|x\|+\|y\|
$$


for all $x, y \in X$, we get

$$
\begin{aligned}
\left\|C_{1}^{P}\left(f_{1}, \ldots, f_{5}\right)\right\|= & \sup _{x, y \in X} e^{-\lambda(\|x\|+\|y\|)} \| f_{1}(x+y)+f_{2}(x-y)-m f_{3}\left(\frac{1}{m} x+y\right) \\
\leq & \quad-m f_{4}\left(\frac{1}{m} x-y\right)-2 m\left(m^{2}-1\right) f_{5}\left(\frac{1}{m} x\right) \| \\
& +|m| \sup _{x, y \in X} e^{-\lambda\|x+y\|}\left\|f_{1}(x+y)\right\|+\sup _{x, y \in X} e^{-\lambda\|x-y\|}\left\|f_{2}(x-y)\right\| \\
& +|m| \sup _{x, y \in X} e^{-\lambda\|(1 / m) x+y\|}\left\|f_{3}\left(\frac{1}{m} x+y\right)\right\| \\
& +2|m|\left(m^{2}-1\right) \sup _{x \in X} e^{-\lambda\|(1 / m) x\|}\left\|f_{4}\left(\frac{1}{m} x-y\right)\right\| \\
= & \left.\left\|f_{1}\right\|+\| \frac{1}{m} x\right) \| \\
\leq & \left(2|m|^{3}+2\right) \max \left\{\left\|f_{1}\right\|,\left\|f_{2}\right\|,\left\|f_{3}\right\|,\left\|f_{4}\right\|,\left\|f_{5}\right\|\right\} \\
= & \left(2|m|^{3}+2\right)\left\|\left(f_{1}, f_{2}, f_{3}, f_{4}, f_{5}\right)\right\|
\end{aligned}
$$

for each $\left(f_{1}, \ldots, f_{5}\right) \in Z_{\lambda}^{5}$. This implies that

$$
\left\|C_{1}^{P}\right\| \leq 2|m|^{3}+2
$$

Now, let $v \in Y$ be such that $\|v\|=1$ and let $\left\{\xi_{n}\right\}_{n}$ be a sequence of positive real numbers decreasing to 0 . We define

$$
f_{n}(x)= \begin{cases}e^{2 \lambda \xi_{n}} v_{,} & \text {if }\|x\|=2 \xi_{n} \text { or }\|x\|=0 \\ -\frac{|m|}{m} e^{2 \lambda \xi_{n}} \mathcal{v}, & \text { if }\|m x\|=|m+1| \xi_{n},\|m x\|=|m-1| \xi_{n} \text { or }\|m x\|=\xi_{n} \\ 0, & \text { otherwise }\end{cases}
$$

for all $x \in X$. Hence we have

$$
e^{-\lambda\|x\|}\left\|f_{n}(x)\right\|= \begin{cases}e^{2 \lambda \xi_{n}}, & \text { if }\|x\|=0, \\ 1, & \text { if }\|x\|=2 \xi_{n} \\ e^{(2-|(m+1) / m|) \lambda \xi_{n},}, & \text { if }\|m x\|=|m+1| \xi_{n}, \\ e^{(2-|(m-1) / m|) \lambda \xi_{n}}, & \text { if }\|m x\|=|m-1| \xi_{n}, \\ e^{(2-1 /|m|) \lambda \xi_{n}}, & \text { if }\|m x\|=\xi_{n}, \\ 0, & \text { otherwise }\end{cases}
$$


for all $x \in X$, so that $f_{n} \in X_{\curlywedge}$ for all positive integers $n$, with

$$
\left\|f_{n}\right\|=e^{2 \lambda \xi_{n}}
$$

Let $u \in X$ be such that $\|u\|=1$ and take $x_{0}, y_{0} \in X$ as $x_{0}=y_{0}=\xi_{n} u$. Then it follows from the definition of $f_{n}$ that

$$
\begin{aligned}
\left\|C_{1}^{P}\left(f_{n}, \ldots, f_{n}\right)\right\| & =\sup _{x, y \in X} e^{-\lambda(\|x\|+\|y\|)} \| f_{n}(x+y)+f_{n}(x-y)-m f_{n}\left(\frac{1}{m} x+y\right) \\
& \quad-m f_{n}\left(\frac{1}{m} x-y\right)-2 m\left(m^{2}-1\right) f_{n}\left(\frac{1}{m} x\right) \| \\
& \geq e^{-2 \lambda \xi_{n}}\left\|e^{2 \lambda \xi_{n}} v+e^{2 \lambda \xi_{n}} v+|m| e^{2 \lambda \xi_{n}} v+|m| e^{2 \lambda \xi_{n}} v+2|m|\left(m^{2}-1\right) e^{2 \lambda \xi_{n}} v\right\| \\
& =2|m|^{3}+2 .
\end{aligned}
$$

If on the contrary $\left\|C_{1}^{P}\right\|<2|m|^{3}+2$, then there exists a $\delta>0$ such that

$$
\left\|C_{1}^{P}\left(f_{n}, \ldots, f_{n}\right)\right\| \leq\left(2|m|^{3}+2-\delta\right)\left\|\left(f_{n}, \ldots, f_{n}\right)\right\|
$$

for all positive integers $n$. So it follows from (2.7), (2.8), and (2.9) that

$$
\left.2|m|^{3}+2 \leq \| C_{1}^{P} f_{n}, \ldots, f_{n}\right) \| \leq\left(2|m|^{3}+2-\delta\right) e^{2 \lambda \xi_{n}}
$$

for all positive integers $n$. Since $\lim _{n \rightarrow \infty} e^{2 \lambda \xi_{n}}=1$, the right-hand side of (2.10) tends to $2|m|^{3}+$ $2-\delta$ as $n \rightarrow \infty$, whence $2|m|^{3}+2 \leq 2|m|^{3}+2-\delta$, which is a contradiction. Hence we have $\left\|C_{1}^{P}\right\|=2|m|^{3}+2$.

Theorem 2.1 of [4] is a result of Theorem 2.1 for $m=2$.

Corollary 2.2. The operator $C_{1}: X_{\curlywedge} \rightarrow X_{\lambda}^{2}$ is a bounded linear operator with

$$
\left\|C_{1}\right\|=2|m|^{3}+2
$$

Proof. The result follows from the proof of Theorem 2.1.

Theorem 2.3. The operator $C_{2}^{P}: Z_{\lambda}^{5} \rightarrow X_{\lambda}^{2}$ is a bounded linear operator with

$$
\left\|C_{2}^{P}\right\|=2|a+b|(a-b)^{2}+|a b(a+b)|+2 .
$$

Proof. Since

$$
\max \left\{\left\|\frac{1}{a} x+\frac{1}{b} y\right\|,\left\|\frac{1}{b} x+\frac{1}{a} y\right\|,\left\|\frac{1}{a b} x\right\|,\left\|\frac{1}{a b} y\right\|,\left\|\frac{1}{a b} x+\frac{1}{a b} y\right\|\right\} \leq\|x\|+\|y\|
$$


for all $x, y \in X$, we get

$$
\begin{aligned}
& \left\|C_{2}^{P}\left(f_{1}, \ldots, f_{5}\right)\right\|=\sup _{x, y \in X} e^{-\lambda(\|x\|+\|y\|)} \| f_{1}\left(\frac{1}{a} x+\frac{1}{b} y\right)+f_{2}\left(\frac{1}{b} x+\frac{1}{a} y\right) \\
& -(a+b)(a-b)^{2}\left[f_{3}\left(\frac{1}{a b} x\right)+f_{4}\left(\frac{1}{a b} y\right)\right] \\
& -a b(a+b) f_{5}\left(\frac{1}{a b} x+\frac{1}{a b} y\right) \| \\
& \leq \sup _{x, y \in X} e^{-\lambda\|(1 / a) x+(1 / b) y\|}\left\|f_{1}\left(\frac{1}{a} x+\frac{1}{b} y\right)\right\| \\
& +\sup _{x, y \in X} e^{-\lambda\|(1 / b) x+(1 / a) y\|}\left\|f_{2}\left(\frac{1}{b} x+\frac{1}{a} y\right)\right\| \\
& +|a+b|(a-b)^{2} \sup _{x \in \mathrm{X}} e^{-\lambda(\|(1 / a b) x\|)}\left\|f_{3}\left(\frac{1}{a b} x\right)\right\| \\
& +|a+b|(a-b)^{2} \sup _{y \in X} e^{-\lambda(\|(1 / a b) y\|)}\left\|f_{4}\left(\frac{1}{a b} y\right)\right\| \\
& +|a b(a+b)| \sup _{x, y \in X} e^{-\lambda\|(1 / a b) x+(1 / a b) y\|}\left\|f_{5}\left(\frac{1}{a b} x+\frac{1}{a b} y\right)\right\| \\
& \leq\left\|f_{1}\right\|+\left\|f_{2}\right\|+|a+b|(a-b)^{2}\left(\left\|f_{3}\right\|+\left\|f_{4}\right\|\right)+|a b(a+b)|\left\|f_{5}\right\| \\
& \leq\left(2|a+b|(a-b)^{2}+|a b(a+b)|+2\right) \max \left\{\left\|f_{1}\right\|,\left\|f_{2}\right\|,\left\|f_{3}\right\|,\left\|f_{4}\right\|,\left\|f_{5}\right\|\right\} \\
& =\left(2|a+b|(a-b)^{2}+|a b(a+b)|+2\right)\left\|\left(f_{1}, f_{2}, f_{3}, f_{4}, f_{5}\right)\right\|
\end{aligned}
$$

for each $\left(f_{1}, \ldots, f_{5}\right) \in Z_{\lambda}^{5}$. This implies that

$$
\left\|C_{2}^{P}\right\| \leq 2|a+b|(a-b)^{2}+|a b(a+b)|+2 .
$$

Let $\eta$ be a real number such that

$$
\eta \notin\left\{0,1, \frac{1-a}{b}, \frac{1-b}{a}, \frac{a-1}{1-b}, \frac{b-1}{1-a}, \frac{a}{1-b}, \frac{b}{1-a}\right\} .
$$

Now, let $u \in X, v \in Y$ be such that $\|u\|=\|v\|=1$ and let $\left\{\xi_{n}\right\}_{n}$ be a sequence of positive real numbers decreasing to 0 . We define

$$
f_{n}(x)= \begin{cases}e^{\lambda(1+|\eta|) \xi_{n}} v, & \text { if } x=\left(\frac{1}{a}+\frac{\eta}{b}\right) \xi_{n} u, \text { or } x=\left(\frac{1}{b}+\frac{\eta}{a}\right) \xi_{n} u, \\ -\frac{|a+b|}{a+b} e^{\lambda(1+|\eta|) \xi_{n}} v, & \text { if } x=\frac{1}{a b} \xi_{n} u, \text { or } x=\frac{\eta}{a b} \xi_{n} u, \\ -\frac{|a b(a+b)|}{a b(a+b)} e^{\lambda(1+|\eta|) \xi_{n}} \mathcal{v}, & \text { if } x=\frac{1+\eta}{a b} \xi_{n} u, \\ 0, & \text { otherwise }\end{cases}
$$


for all $x \in X$. Hence we have

$$
e^{-\lambda\|x\|}\left\|f_{n}(x)\right\|= \begin{cases}e^{(1+|\eta|-|1 / a+\eta / b|) \lambda \xi_{n}}, & \text { if } x=\left(\frac{1}{a}+\frac{\eta}{b}\right) \xi_{n} u, \\ e^{(1+|\eta|-|1 / b+\eta / a|) \lambda \xi_{n}}, & \text { if } x=\left(\frac{1}{b}+\frac{\eta}{a}\right) \xi_{n} u, \\ e^{(1+|\eta|-|1 / a b|) \lambda \xi_{n},} & \text { if } x=\frac{1}{a b} \xi_{n} u, \\ e^{(1+|\eta|-|\eta / a b|) \lambda \xi_{n},} & \text { if } x=\frac{\eta}{a b} \xi_{n} u, \\ e^{(1+|\eta|-|(1+\eta) / a b|) \lambda \xi_{n}}, & \text { if } x=\frac{1+\eta}{a b} \xi_{n} u, \\ 0, & \text { otherwise }\end{cases}
$$

for all $x \in X$, so that $f_{n} \in X_{\mathcal{l}}$ for all positive integers $n$, with

$$
\begin{aligned}
\left\|f_{n}\right\|=\max \left\{e^{(1+|\eta|-|1 / a+\eta / b|) \lambda \xi_{n}}, e^{(1+|\eta|-|1 / b+\eta / a|) \lambda \xi_{n}},\right. \\
\left.\quad e^{(1+|\eta|-|1 / a b|) \lambda \xi_{n}}, e^{(1+|\eta|-|\eta / a b|) \lambda \xi_{n}}, e^{(1+|\eta|-|(1+\eta) / a b|) \lambda \xi_{n}}\right\} .
\end{aligned}
$$

Let $x_{0}, y_{0} \in X$ be such that $x_{0}=\xi_{n} u$ and $y_{0}=\eta \xi_{n} u$. Then it follows from the definition of $f_{n}$ that

$$
\begin{aligned}
\left\|C_{2}^{P}\left(f_{n}, \ldots, f_{n}\right)\right\| & =\sup _{x, y \in X} e^{-\lambda(\|x\|+\|y\|)} \| \\
& -(a+b)(a-b)^{2}\left[f_{n}\left(\frac{1}{a} x+\frac{1}{b} y\right)+f_{n}\left(\frac{1}{a b} y\right)\right] \\
& -a b(a+b) f_{n}\left(\frac{1}{a b} x+\frac{1}{a b} y\right) \| \\
\geq & e^{-\lambda(1+|\eta|) \xi_{n}}\left\|e^{\lambda(1+|\eta|) \xi_{n}}+e^{\lambda(1+|\eta|) \xi_{n}}+2|a+b|(a-b)^{2} e^{\lambda(1+\eta \eta \mid) \xi_{n}}+|a b(a+b)| e^{\lambda(1+\eta \eta \mid) \xi_{n}}\right\| \\
& =2|a+b|(a-b)^{2}+|a b(a+b)|+2,
\end{aligned}
$$

so that

$$
\left\|C_{2}^{P}\left(f_{n}, \ldots, f_{n}\right)\right\| \geq 2|a+b|(a-b)^{2}+|a b(a+b)|+2 .
$$

If on the contrary $\left\|C_{2}^{P}\right\|<2|a+b|(a-b)^{2}+|a b(a+b)|+2$, then there exists a $\delta>0$ such that

$$
\left\|C_{2}^{P}\left(f_{n}, \ldots, f_{n}\right)\right\| \leq\left(2|a+b|(a-b)^{2}+|a b(a+b)|+2-\delta\right)\left\|\left(f_{n}, \ldots, f_{n}\right)\right\|
$$


for all positive integers $n$. So it follows from (2.21) and (2.22) that

$$
2|a+b|(a-b)^{2}+|a b(a+b)|+2 \leq\left\|C_{2}^{P}\left(f_{n}, \ldots, f_{n}\right)\right\| \leq\left(2|a+b|(a-b)^{2}+|a b(a+b)|+2-\delta\right)\left\|f_{n}\right\|
$$

for all positive integers $n$. Since $\lim _{n \rightarrow \infty} \xi_{n}=0$, it follows from (2.19) that $\lim _{n \rightarrow \infty}\left\|f_{n}\right\|=1$, so the right-hand side of (2.23) tends to $2|a+b|(a-b)^{2}+|a b(a+b)|+2-\delta$ as $n \rightarrow \infty$, whence

$$
2|a+b|(a-b)^{2}+|a b(a+b)|+2 \leq 2|a+b|(a-b)^{2}+|a b(a+b)|+2-\delta,
$$

which is a contradiction. Hence we have $\left\|C_{2}^{P}\right\|=2|a+b|(a-b)^{2}+|a b(a+b)|+2$.

Corollary 2.4. The operator $C_{2}: X_{\curlywedge} \rightarrow X_{\lambda}^{2}$ is a bounded linear operator with

$$
\left\|C_{2}\right\|=2|a+b|(a-b)^{2}+|a b(a+b)|+2
$$

Proof. The result follows from the proof of Theorem 2.3.

\section{Acknowledgment}

The authors would like to thank the referee for his/her useful comments.

\section{References}

[1] S. Czerwik, Functional Equations and Inequalities in Several Variables, World Scientific, River Edge, NJ, USA, 2002.

[2] S. Czerwik and K. Dlutek, "Cauchy and Pexider operators in some function spaces," in Functional Equations, Inequalities and Applications, pp. 11-19, Kluwer Academic Publishers, Dordrecht, The Netherlands, 2003.

[3] M. S. Moslehian, T. Riedel, and A. Saadatpour, "Norms of operators in $X_{\curlywedge}$ spaces," Applied Mathematics Letters, vol. 20, no. 10, pp. 1082-1087, 2007.

[4] S.-M. Jung, "Cubic operator norm on $X_{\curlywedge}$ space," Bulletin of the Korean Mathematical Society, vol. 44, no. 2, pp. 309-313, 2007.

[5] K.-W. Jun and H.-M. Kim, "The generalized Hyers-Ulam-Rassias stability of a cubic functional equation," Journal of Mathematical Analysis and Applications, vol. 274, no. 2, pp. 867-878, 2002.

[6] K.-W. Jun, H.-M. Kim, and I.-S. Chang, "On the Hyers-Ulam stability of an Euler-Lagrange type cubic functional equation," Journal of Computational Analysis and Applications, vol. 7, no. 1, pp. 21-33, 2005.

[7] K.-W. Jun and H.-M. Kim, "On the stability of Euler-Lagrange type cubic mappings in quasi-Banach spaces," Journal of Mathematical Analysis and Applications, vol. 332, no. 2, pp. 1335-1350, 2007. 